\title{
Rosmarinic acid attenuates cardiac fibrosis following long-term pressure overload via AMPKa/Smad3 signaling
}

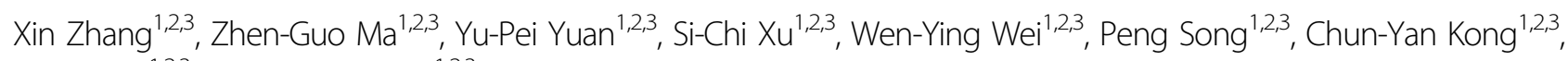
Wei Deng ${ }^{1,2,3}$ and Qi-Zhu Tang ${ }^{1,2,3}$

\begin{abstract}
Agonists of peroxisome proliferator-activated receptor gamma (PPAR- $\gamma$ ) can activate $5^{\prime}$ AMP-activated protein kinase alpha (AMPKa) and exert cardioprotective effects. A previous study has demonstrated that rosmarinic acid (RA) can activate PPAR- $\gamma$, but its effect on cardiac remodeling remains largely unknown. Our study aimed to investigate the effect of RA on cardiac remodeling and to clarify the underlying mechanism. Mice were subjected to aortic banding to generate pressure overload induced cardiac remodeling and then were orally administered RA ( $100 \mathrm{mg} / \mathrm{kg} /$ day) for 7 weeks beginning 1 week after surgery. The morphological examination, echocardiography, and molecular markers were used to evaluate the effects of RA. To ascertain whether the beneficial effect of RA on cardiac fibrosis was mediated by AMPKa, AMPKa2 knockout mice were used. Neonatal rat cardiomyocytes and fibroblasts were separated and cultured to validate the protective effect of RA in vitro. RA-treated mice exhibited a similar hypertrophic response as mice without RA treatment, but had an attenuated fibrotic response and improved cardiac function after pressure overload. Activated AMPKa was essential for the anti-fibrotic effect of RA via inhibiting the phosphorylation and nuclear translocation of Smad3 in vivo and in vitro, and AMPKa deficiency abolished RA-mediated protective effects. Small interfering RNA against Ppar- $\gamma$ (siPpar- $\gamma$ ) and GW9662, a specific antagonist of PPAR- $\gamma$, abolished RA-mediated AMPKa phosphorylation and alleviation of fibrotic response in vitro. RA attenuated cardiac fibrosis following long-term pressure overload via AMPKa/Smad3 signaling and PPAR- $\gamma$ was required for the activation of AMPKa. RA might be a promising therapeutic agent against cardiac fibrosis.
\end{abstract}

\section{Introduction}

Cardiac remodeling, characterized as cardiomyocyte hypertrophy and interstitial fibrosis, is a leading risk factor for heart failure, arrhythmia, and sudden death ${ }^{1,2}$. As a key feature of cardiac remodeling, cardiac fibrosis is defined as superfluous transdifferentiation from cardiac fibroblasts (CFs) to myofibroblasts and a disrupted

\footnotetext{
Correspondence: Wei Deng (vivideng1982@whu.edu.cn) or Q.-Z. Tang (qztang@whu.edu.cn)

${ }^{1}$ Department of Cardiology, Renmin Hospital of Wuhan University, Wuhan 430060, China

${ }^{2}$ Cardiovascular Research Institute of Wuhan University, Wuhan 430060, China Full list of author information is available at the end of the article

Xin Zhang and Zhen-Guo Ma contributed equally to this work.

Edited by S. Lavandero
}

\section{(c) The Author(s). 2018}

homeostasis between synthesis and degradation of the extracellular matrix $(\mathrm{ECM})^{3}$. Once activated, CFs phenoconvert into myofibroblasts and secrete ECM. The ECM provides structural support for cardiomyocytes and nonstructural components under physiological conditions but leads to cardiac fibrosis with anomalous deposition in the presence of chronic injurious stimuli ${ }^{4}$. Massive ECM accumulation may impair mechanical and electrical functions of the heart and subsequently result in heart failure and arrhythmogenesis ${ }^{5}$. Besides, excessive perivascular ECM may also deform the vasculature and separate myocytes from adjacent capillaries, which limits the blood supply of myocytes ${ }^{6}$. CFs are responsible for \footnotetext{
changes were made. The images or other third party material in this article are included in the article's Creative Commons license, unless indicated otherwise in a credit line to the material. If material is not included in the article's Creative Commons license and your intended use is not permitted by statutory regulation or exceeds the permitted use, you will need to obtain permission directly from the copyright holder. To view a copy of this license, visit http://creativecommons.org/licenses/by/4.0/.
} 
orchestrating cardiac fibrosis; thus, effective strategies that targeting CFs may help to develop efficacious interventions against fibrosis, mitigate cardiac remodeling, and postpone the development of heart failure ${ }^{7}$.

Smad3 is identified as a central intracellular mediator of myofibroblasts transdifferentiation and ECM synthesis in the pathogenesis of cardiac fibrosis ${ }^{8,9}$. A previous study indicated that knocking out Smad3 in mice blocked myofibroblasts activation and protected against angiotensin II-induced cardiac fibrosis ${ }^{10}$. Enhanced phosphorylation and nuclear translocation of Smad3 in fibroblasts was largely responsible for the aggravating fibrosis phenotype following myocardial infarction ${ }^{8}$. These results indicated that inhibition of Smad3 might be beneficial for alleviating cardiac fibrosis and that unearthing a negative regulator of Smad3 could be of paramount clinical importance. $5^{\prime}$ AMP-activated protein kinase (AMPK) has been recognized as a key regulator of energy metabolism in the heart; however, current available studies implied that AMPK extended well beyond its energy-regulating function and played a crucial role in regulating cardiac fibrosis $^{11,12}$. AMPK deficiency exacerbated transverse aortic constriction-induced cardiac fibrosis, whereas the activation of AMPK attenuated cardiac fibrosis ${ }^{11,13}$. Moreover, a previous study indicated that the activation of AMPK negatively regulated Smad3 activation and exerted anti-fibrotic effects ${ }^{14}$. Therefore, pharmacological activation of AMPK may be of great therapeutic interest for treating cardiac fibrosis.

Rosmarinic acid ( $\alpha$-o-caffeoyl-3, 4-dihydroxyphenyl lactic acid; RA), a natural poly-phenolic compound, is widely distributed in species of the Boraginaceae and subfamily Nepetoideae of the Labiatae, which include oregano, sage, mint, sweet basil, and perilla, etc ${ }^{15,16}$. Growing interests are raised on RA for its wide spectrum of biological activities, delineated as powerful anti-oxidative, anti-inflammatory, anti-proliferative, anti-bacterial, and human immunodeficiency virus-1-inhibiting properties, etc ${ }^{17-19}$. RA has been reported to regulate mitogen-activated protein kinase (MAPK) and protein kinase B (PKB/AKT) signaling, which are critically involved in cardiac hypertrophy and fibrosis ${ }^{16,20,21}$. Besides, RA could suppress hypertension via regulating angiotensin-converting enzyme ${ }^{22}$ or the endothelium-dependent vasodilator effect $^{23}$. It is noteworthy that RA could activate peroxisome proliferatoractivated receptor gamma (PPAR- $\gamma$ ) in hepatic stellate cells and exerted anti-fibrotic effect ${ }^{24}$. Previous studies indicated that agonists of PPAR- $\gamma$ could be also effective in activating AMPK $^{25,26}$. Based on these findings, we hypothesized that RA may be a promising candidate for the treatment of cardiac hypertrophy and fibrosis.

In the current study, we provided the first evidence of the protective effects and underlying mechanisms of RA on cardiac hypertrophy and fibrosis. We found that RA could attenuate cardiac fibrosis in mice induced by pressure overload with no alteration in hypertrophic phenotype. Mechanistically, we demonstrated that the protective effects of RA on cardiac fibrosis may be attributed to its activation of AMPK $\alpha$ and inhibition of Smad3 in vivo and in vitro.

\section{Results}

\section{RA attenuated cardiac dysfunction in mice following} long-term pressure overload

To clarify whether the beneficial effect of RA on cardiac dysfunction is secondary to its anti-hypertensive effects, we first investigated the blood pressure and found no difference in the maximum carotid artery pressure between vehicle and RA-treated group either at baseline or after the aortic banding $(\mathrm{AB})$ operation (Fig. 1a). Mice exhibited cardiac dysfunction with reduced fractional shortening (FS) and $\pm \mathrm{dp} / \mathrm{dt}$ in response to 8 -week pressure overload, which were significantly attenuated after RA treatment (Figs. 1bd). No alteration in body weight $(\mathrm{BW})$ or heart rate was observed among all groups (Figure S1a-b). We next examined the potential effect of RA on cardiac hypertrophy and observed similar hypertrophic responses in mice with or without RA treatment, as revealed by the similar increases in heart weight (HW)/BW, HW/tibia length (TL), and interventricular septal thickness at systole or diastole (IVSs or IVSd) (Figs. 1e-h). All the echo data are shown in Supplementary Table 1. This observation was further confirmed by the hematoxylin and eosin (HE) staining and cross-sectional area of cardiomyocytes (Figs. 1i, j). In line with these data, the mRNA levels of markers associated with cardiac hypertrophy, including Anp and $\beta$-Mhc, showed no significant alterations with or without RA treatment, despite the downregulated mRNA level of brain natriuretic peptide (Bnp) was observed in $A B+R A$ group compared with that of $A B$ group (Figure S1c-d, Fig. 1k). Taken together, these results indicated that RA could attenuate cardiac dysfunction in mice following long-term pressure overload independent of cardiac hypertrophy inhibition.

\section{RA protected against cardiac fibrosis in vivo}

As cardiac fibrosis is an essential feature of the cardiac remodeling, and contributes to the conversion from hypertrophy to heart failure by increasing myocardial stiffness and reducing pumping capability, ${ }^{3,5}$, we thus detected the effect of RA on cardiac fibrosis. As shown in Figs. 2a, b, RA-treated mice presented with less collagen deposition than that in mice without RA treatment after $A B$ insult, which came in parallel with reduced mRNA levels of fibrotic markers, collagen I (Col I), Col III, connective tissue growth factor (Ctgf), fibronectin (Fn), transforming growth factor-beta (Tgf- $\beta 1$ ) and $\alpha$-smooth muscle actin ( $\alpha$-Sma; Figs. 2c-h). Cardiac fibrosis was 


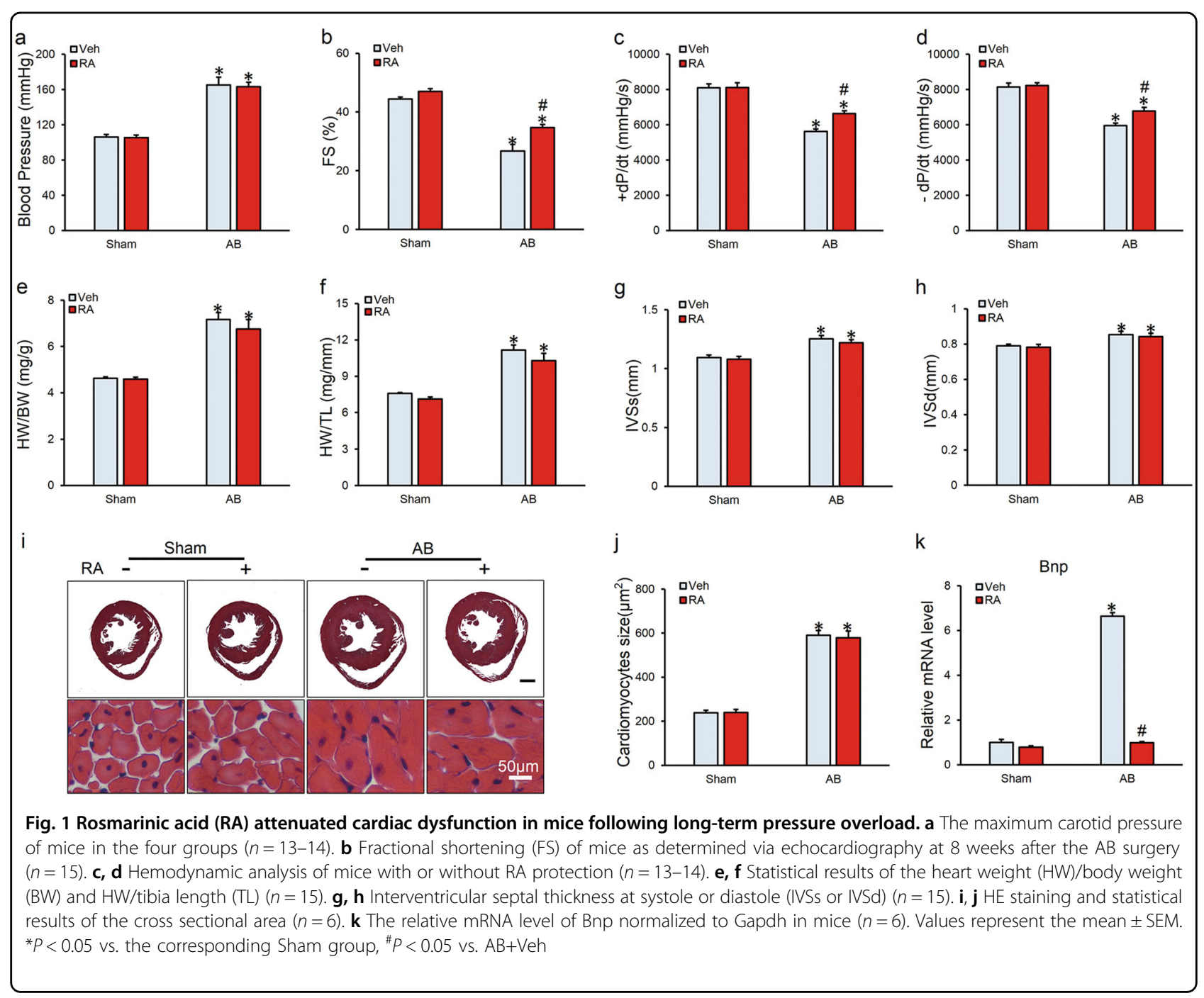

further quantified by immunochemistry of $\alpha$-SMA and we found that RA treatment decreased the expression of $\alpha$ SMA in hypertrophic hearts (Figs. 2a, b).

Seeing that oxidative stress and inflammation are implicated in the pathogenesis of cardiac fibrosis ${ }^{27,28}$ and RA has been reported to blunt oxidative stress and inflammation via NF-E2-related factor 2 (Nrf2), inducible nitric oxide synthase (iNOS) and cyclooxygenase- 2 (COX2) signaling ${ }^{29-31}$, we speculated whether the cardioprotective effect of RA was secondary to alleviated oxidative stress and inflammation. No change in nuclear translocation of Nrf2 or superoxide dismutase 2 (SOD2) expression was observed in mice with or without RA treatment (Figure S2a-c). We previously found that inflammatory response almost returned to normal level at 8 weeks after $\mathrm{AB}^{32}$, thus we examined the effect of RA on inflammation 4 weeks after surgery. Unexpectedly, increased expression levels of COX2 and iNOS after $A B$ surgery were unaffected by RA (Figure S2a, d-e), and correspondingly, mRNA level of inflammatory markers exhibited no difference in hypertrophic hearts treated with RA except for Tnf- $\alpha$ (Figure S2f).

\section{RAactivated AMPKa in vivo and knockout of AMPKa abrogated its protective effects}

We further examined the precise mechanism involved in the anti-fibrotic effect of RA. Previous studies indicated that pretreatment with RA could inhibit phosphorylation of MAPKs ${ }^{16,21,33}$ and increase the phosphorylation of $\mathrm{AKT}^{20,34}$, therefore we investigated MAPKs and AKT pathway after RA treatment. Unexpectedly, RA did not alter the phosphorylation of extracellular regulated protein kinases (ERK), P38 or AKT (Figs. 3a-d). Agonists of PPAR- $\gamma$ have been proven to be effective in activating AMPK, and we found that AMPK $\alpha$ was activated by RA in hypertrophic hearts, further validated by increased phosphorylation of ACC, which could reflect a high AMPK $\alpha$ activity (Figs. 3a, e, f). To gain evidence that anti-fibrotic effects of RA were mediated 


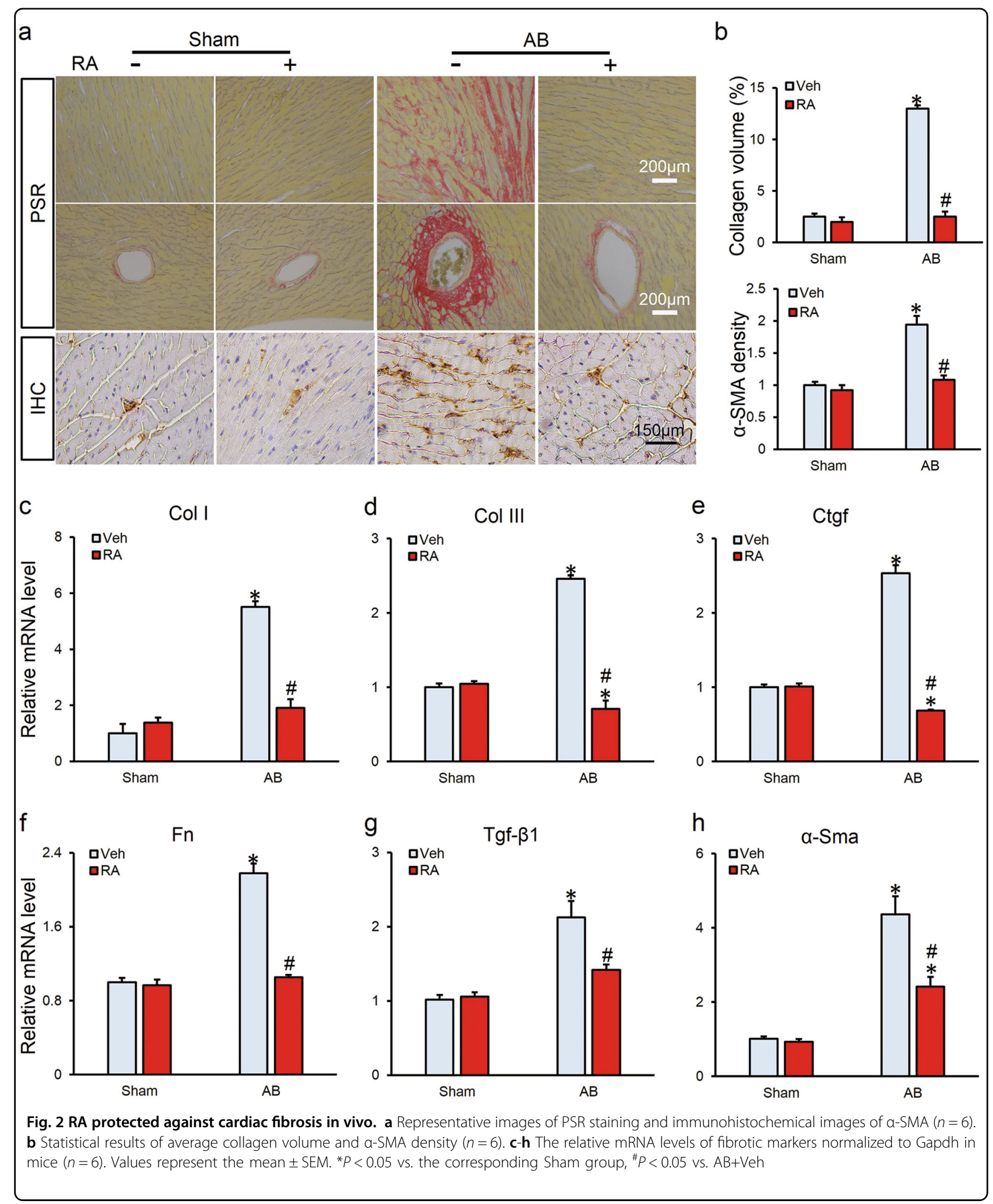




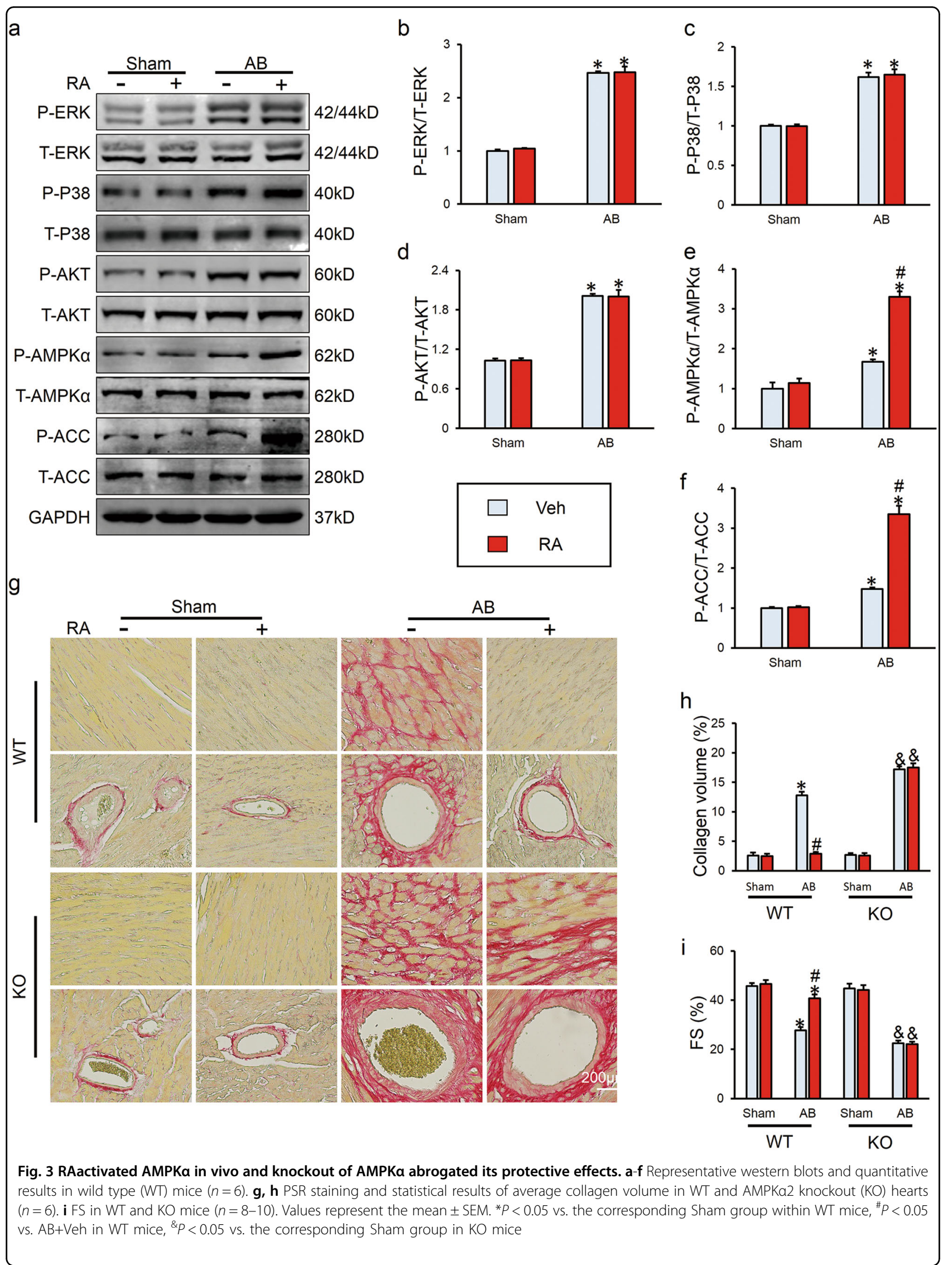


via the activation of $A M P K \alpha$, we used $A M P K \alpha 2$ knockout (KO) mice. As expected, KO of AMPK $\alpha$ abrogated the protective effects of RA on cardiac fibrosis in response to pressure overload, as evidenced by picrosirius red (PSR) staining (Figs. 3g, h). Correspondingly, ameliorated cardiac function after RA treatment was abolished after the absence of AMPK $\alpha$ (Fig. 3i). All the echo data are shown in Supplementary Table 2.

\section{RA suppressed Smad3 phosphorylation and nuclear translocation through AMPKa activation}

Intensive investigation is underway to shed light into the mechanisms of RA in ameliorating cardiac fibrosis. It is well recognized that Smad3 is a critical and necessary mediator of cardiac fibrosis in response to pressure over$\operatorname{load}^{8,35}$, we then examined the effect of RA on the phosphorylation and nuclear translocation of Smad3, which are important for determining the strength and duration of the signal and biological response ${ }^{8,36}$. Increased phosphorylation of Smad3 was observed after pressure overload, which was prevented after the treatment of RA (Figs. 4a, b). After sub-fractionating left ventricles into cytosolic fraction and nuclear fraction, we found that increased protein level of Smad3 in nuclear fraction and decreased level in cytosolic fraction after $A B$ operation were reversed by RA (Figs. 4a, c). RA alone showed no influence on phosphorylation or nuclear translocation of Smad3 in sham surgery (Fig. 4a-c). And previous study showed that activation of AMPK $\alpha$ could inhibit phosphorylation and nuclear translocation of Smad $3^{14}$, we further investigated that whether AMPK $\alpha$ was involved in the regulation on Smad3 of RA, western blot analysis showed that inhibitory effect of RA on Smad3 was counteracted in AMPK $\alpha 2 \mathrm{KO}$ mice (Figs. $4 \mathrm{a}-\mathrm{c}$ ).

\section{RA blocked transdifferentiation of CFs via AMPKa/ Smad3 signaling pathway in vitro}

There is common agreement that CFs are the primary cell type most responsible for cardiac fibrosis during pathological cardiac remodeling and transdifferentiation of CFs to myofibroblasts is a hallmark in cardiac fibrosis. To further assess the effect of RA in vitro, we separated neonatal rat CFs and cardiomyocytes. Consistent with the data in vivo, we found unaffected cross sectional area and undiminished mRNA level of hypertrophic markers after RA treatment $(20 \mu \mathrm{g} / \mathrm{ml})$ in terms of cardiomyocytes in response to phenylephrine (PE) stimuli (Figure S3a-d). As shown in Figs. $5 \mathrm{a}, \mathrm{b}, \mathrm{RA}$ inhibited TGF- $\beta$-induced mRNA expression of fibrillar collagen in a dose-dependent manner. Mechanistically, continuous activation of AMPK $\alpha$ in a dose-dependent manner by RA was also observed (Fig. 5d). As the anti-fibrotic effect mediated by RA may be ascribed to its cell damage activity ${ }^{37}$, thus we tested the viability of CFs and no cytotoxicity was found in the concentration gradient used in our experiment (Fig. 5c). RA $(30 \mu \mathrm{g} / \mathrm{ml})$ decreased the mRNA level of fibrotic markers and the expression of $\alpha$-SMA, all of which were negated after the knockdown of AMPK $\alpha 2$ (Figs. 5e, f, Figure S3e-g). In addition, RA treatment significantly inhibited TGF- $\beta$-induced CFs migration and the effect was blocked by AMPK inhibition (Fig. 5g). Our results further confirmed that RA reversed the phosphorylation and nuclear translocation of Smad3, which was blocked in the absence of AMPK $\alpha 2$ (Figs. 6a-c). These results showed that RA blocked transdifferentiation of CFs via $A M P K \alpha / S m a d 3$ signaling pathway in vitro, and subsequently decreased the accumulation of ECM.
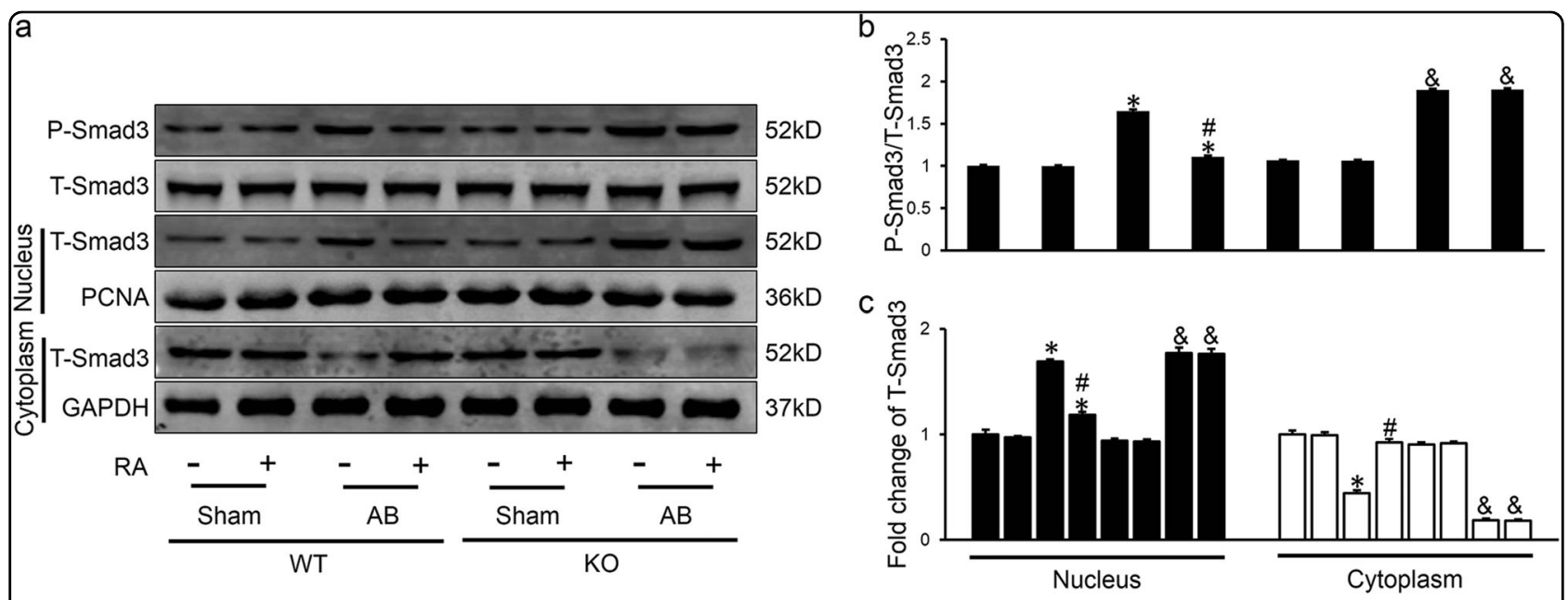

Fig. 4 RA suppressed Smad3 phosphorylation and nuclear translocation through AMPKa activation. a-c Representative western blots and statistical results $(n=6)$. Values represent the mean \pm SEM. ${ }^{*} P<0.05$ vs. the corresponding Sham group within WT mice, ${ }^{\#} P<0.05$ vs. AB+Veh in $W T$ mice, ${ }^{\circledR} P<0.05$ vs. the corresponding Sham group in $\mathrm{KO}$ mice 


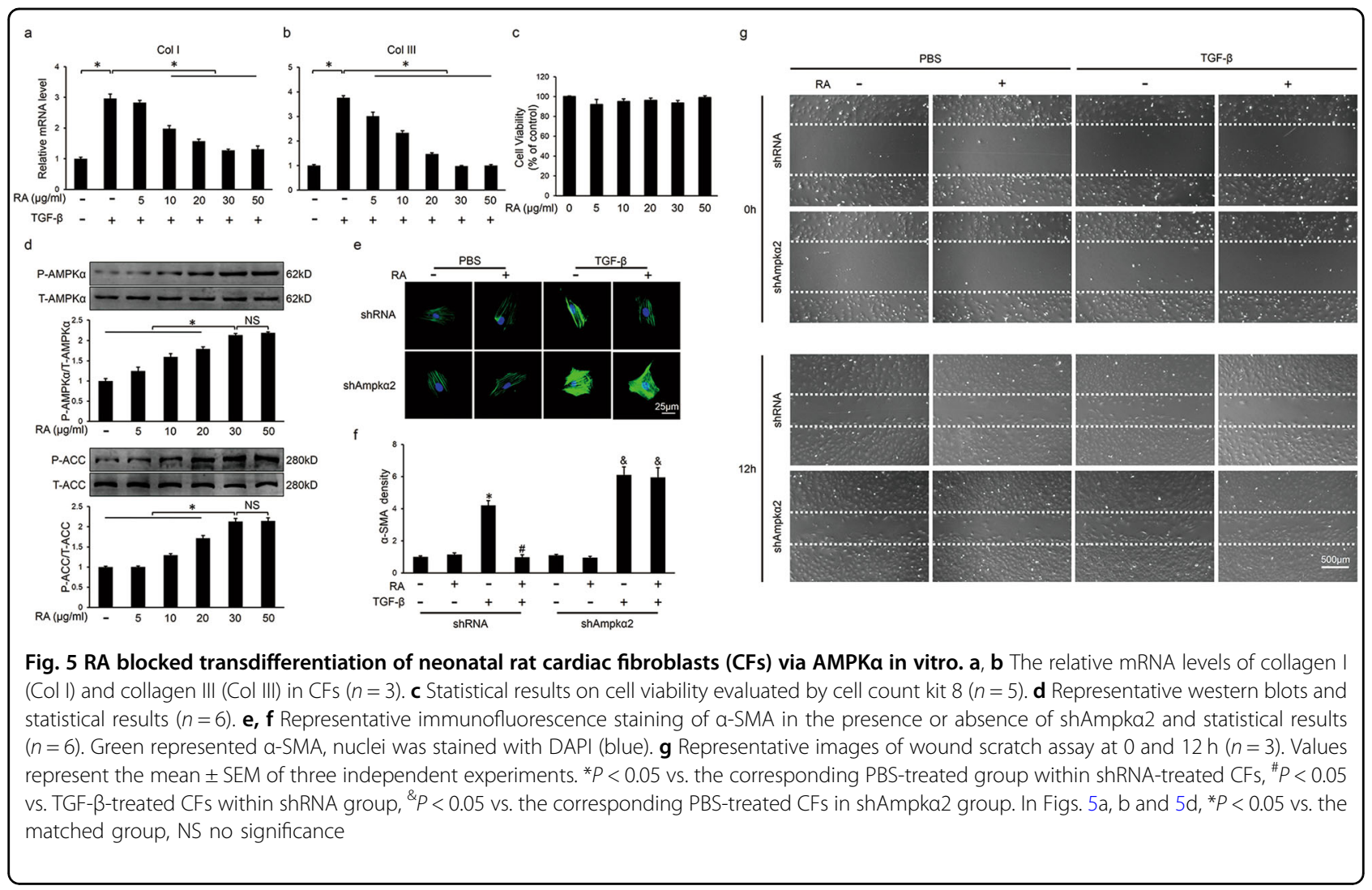

\section{RAactivated AMPKa via the activation of PPAR- $\gamma$}

Finally, we investigated the possible mechanism by which RA-activated AMPK $\alpha$. Previous study showed that RA could epigenetically de-repress Ppar- $\gamma$ in hepatic stellate cells for the anti-fibrotic effect ${ }^{24}$, and we observed that mRNA and protein levels of PPAR- $\gamma$ were decreased after $A B$, which were restored after RA treatment in vivo (Fig. 7a, Figure S4a). In addition, RA increased mRNA level and protein expression of PPAR- $\gamma$ in a dosedependent manner in vitro, which aligns with AMPK $\alpha$ activation (Figs. 5d, 7b, Figure S4b). To evaluate the necessity of PPAR- $\gamma$ in RA-activated AMPK $\alpha$, we treated CFs with GW9662, and found that activation of AMPK $\alpha$ was inhibited (Fig. 7c), followed by an aggravating fibrotic response (Figs. 7d-f). Knockdown of PPAR- $\gamma$ with small interfering RNA against Ppar- $\gamma$ (siPpar- $\gamma$ ) further confirmed our hypothesis (Figs. 7g, h, Figure S4c). Thus, we concluded that RA might activate AMPK $\alpha$ via the activation of PPAR- $\gamma$.

\section{Discussion}

In the present study, we found that RA attenuated cardiac dysfunction and cardiac fibrosis following longterm pressure overload. Supplementation of RA blocked transdifferentiation of CFs and inhibited accumulation of collagen in TGF- $\beta$-treated CFs. AMPK $\alpha$ suppressed phosphorylation and nuclear translocation of Smad3, and these protective effects were abolished by AMPK $\alpha$ deficiency in vivo and in vitro. And we also found that PPAR$\gamma$ was essential for the activation of AMPK $\alpha$. Thus, our current findings identified RA as a novel therapeutic agent against cardiac fibrosis.

Increasing pieces of evidence indicated a possible role for RA on cardiac remodeling due to its pleiotropic bioactivities, including powerful anti-oxidative and antiinflammatory effects ${ }^{19,31,38}$. RA could decrease oxidative stress-induced cell damage via activating Nrf2/antioxidant response element signaling pathway ${ }^{31}$. The inhibitory effect on nuclear factor kappa-light chain-enhancer of activated $B$ cells activation could reduce inflammation response $^{39}$. However, our study demonstrated that RA exerted a protective effect on cardiac fibrosis independent of the aforementioned factors. Obviously, excessive deposition of ECM results in profound structural and functional abnormalities of the heart. Clinical researches disclosed that late gadolinium enhancement defined fibrosis was associated with occurrence of sudden cardiac death, high rate of future cardiovascular event, and heart failure hospitalization ${ }^{40,41}$, suggesting a deleterious role of cardiac fibrosis and an urgent need for pharmacological interventions. The currently available pharmacological treatment for cardiac fibrosis, including agents targeting 


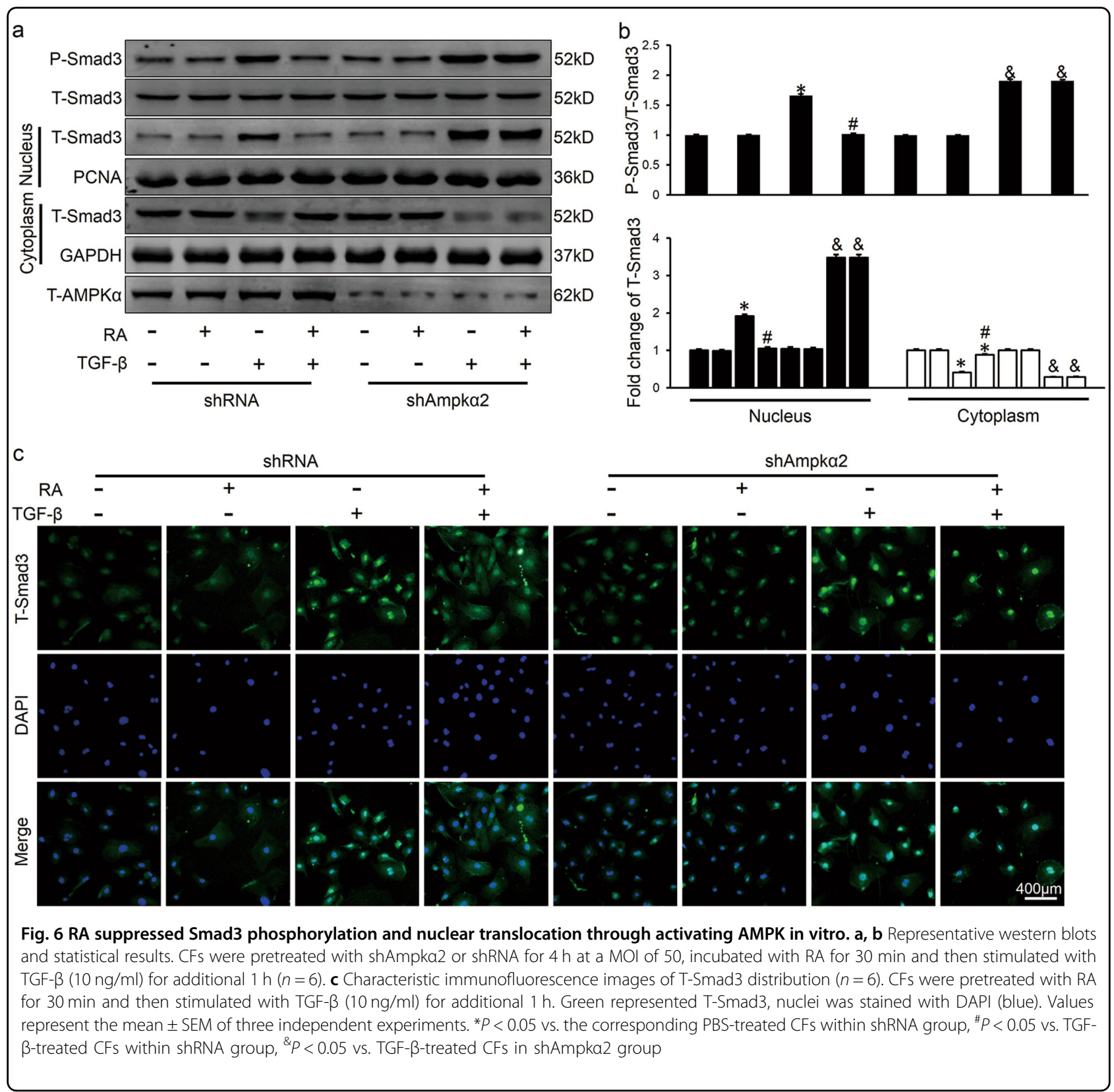

renin-angiotensin-aldosterone system or sympathetic nerves system, achieved certain effects, but their applications are limited in some clinical conditions due to their side effects. Thus, it is of great significance that our results collectively provided RA as an anti-fibrotic candidate in the prevention and treatment of cardiac fibrosis.

AMPK is a highly conserved eukaryotic serine/threonine protein kinase, mainly known as an energy sensor in regulating energy homeostasis; however, current available studies showed that its actions extended well beyond its energy-regulating function and it appeared to play an essential role in regulating cardiac fibrosis ${ }^{42,43}$. Activation of AMPK led to suppressed cardiac fibrosis and improved cardiac function ${ }^{12,44}$, whereas deletion of AMPK was detrimental for cardiac function because of the aggravated fibrosis ${ }^{11}$. And our previous study also indicated that activation of AMPK prevented pressure overload induced cardiac fibrosis ${ }^{45}$. Herein, we found that RA prevented against cardiac fibrosis via activating AMPK and KO of AMPK abrogated the protective effect, which is consistent with a previous study showing that RA-triggered activation of AMPK was responsible for its inhibition on insulin resistance in skeletal muscle cells ${ }^{46}$. The data in our study indicated a key role of AMPK in RA-mediated cardiac protection. 


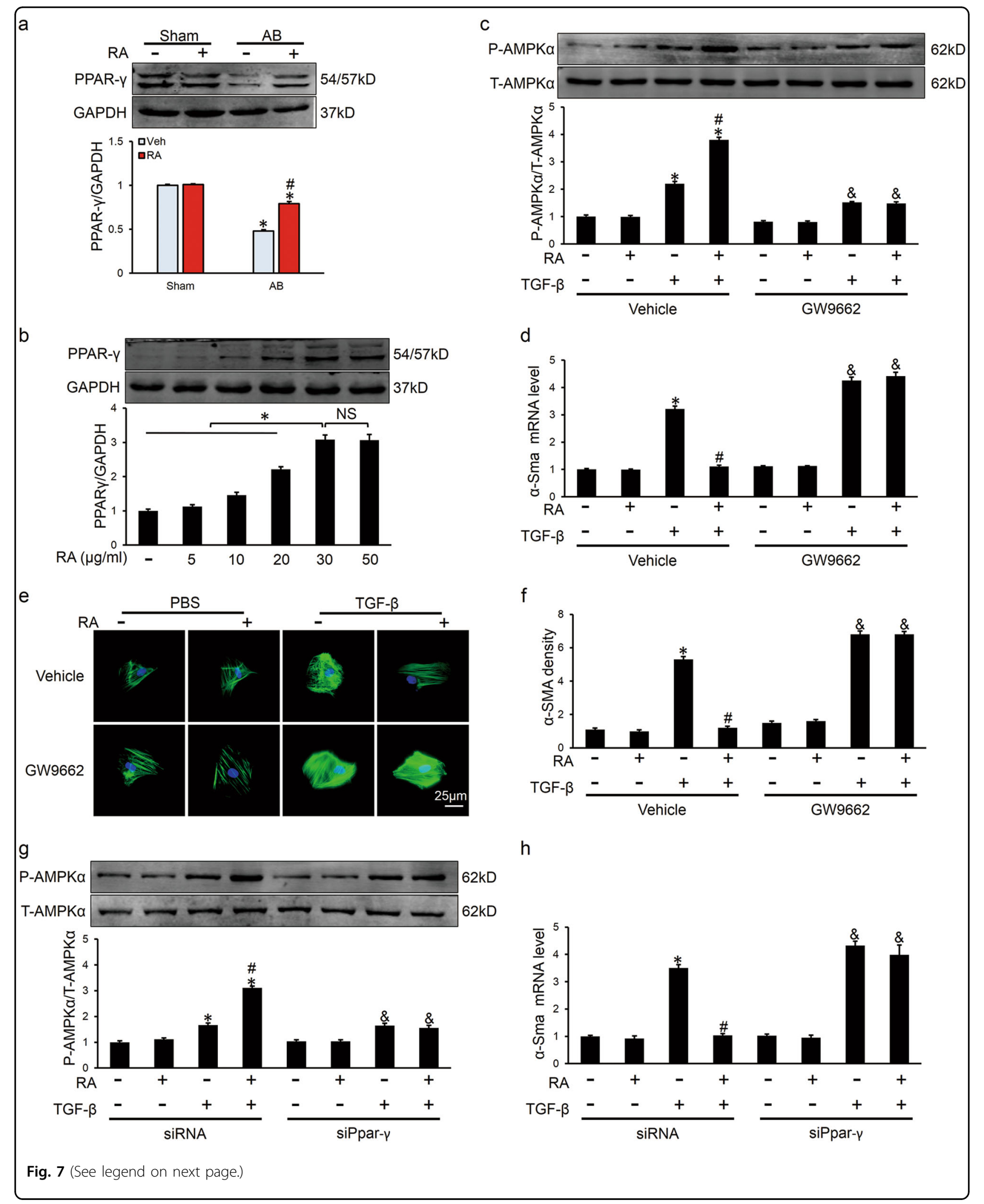


(see figure on previous page)

Fig. 7 RAactivated AMPKa via the activation of PPAR- - . a The expression of PPAR- $\gamma$ and the quantitative results in vivo $(n=6)$. $\mathbf{b}$ Representative western blots and statistical results of PPAR- $\gamma$ in CFs $(n=6)$. c Activation of AMPKa was blocked in the presence of GW9662 in CFs ( $n=6)$. d The relative mRNA level of a-Sma normalized to Gapdh in the presence or absence of GW9662 in CFs $(n=6)$. e, $\mathbf{f}$ Representative immunofluorescence staining of a-SMA and statistical results $(n=6)$. Green represented a-SMA, nuclei was stained with DAPI (blue). g Activation of AMPKa was blocked after knockdown of PPAR- $\gamma$ with siPpar- $\gamma$ in CFs $(n=6)$. $\mathbf{h}$ The relative mRNA level of a-Sma normalized to Gapdh with or without siPpar- $\gamma$ treatment in CFs $(n=6)$.Values represent the mean \pm SEM. Representative images are shown in $\mathbf{b}-\mathbf{h}$ from three independent experiments. ${ }^{*} P<0.05$ vs. the corresponding control CFs within vehicle or siRNA group, ${ }^{\#} P<0.05$ vs. TGF- $\beta$-treated CFs within vehicle or shRNA group, ${ }^{\circledR} P<0.05$ vs. the corresponding control CFs within GW9662 or siPpar- $\gamma$ group. In Figs. $7 a,{ }^{*} P<0.05$ vs. the corresponding Sham group, ${ }^{\sharp} P<0.05$ vs. AB+Veh. In Figs. $7 b$, ${ }^{*} P<0.05$ vs. the matched group, NS no significance

Smad3 is a key transcription factor that mediates the pathogenesis of cardiac fibrosis and targeting Smad3 could inhibit the initiation and progression of cardiac fibrosis ${ }^{8}$. Once phosphorylated and activated, Smad3 forms a complex with co-Smad and translocates to the nucleus, where it binds to specific cis-elements and enhances the expression of fibrillar collagen ${ }^{9}$. Huang et al. showed that KO of Smad3 in mice protected against cardiac fibrosis induced by angiotensin II infusion ${ }^{10}$. In the present study, we showed that RA suppressed phosphorylation and nuclear translocation of Smad3 in an AMPK-dependent manner, which is consistent with a previous study ${ }^{14}$. In contrast, Rangnath et al. demonstrated that in TGF- $\beta$-induced human primary mesangial cells and murine embryonic fibroblasts, AMPK blocked Smad3-mediated transcription of fibrosis-related genes by inhibiting its binding to the promoter of the target gene but did not inhibit Smad3 phosphorylation or nuclear translocation ${ }^{47}$. Taken together, we found that the protective effect of RA on cardiac fibrosis was mediated partially, if not fully, through the activation of AMPK and the inhibition of Smad3.

PPAR- $\gamma$, a member of the nuclear receptor superfamily, is regulated by direct binding of steroid and thyroid hormones, vitamins, and lipid metabolites, etc. Recent evidence identified RA as an activator of PPAR- $\gamma$ involving the MeCP2-EZH2 relay via Wnt signaling ${ }^{24}$. We found that PPAR- $\gamma$ was essential for the activation of AMPK by RA, in line with previous data showing that endothelial cell survival and function preservation was relied on PPAR- $\gamma$-dependent activation of $\mathrm{AMPK}^{48}$. PPAR- $\gamma$ is primarily expressed in adipose tissue, where it is the key orchestrator of the transcriptional cascade mediating adipocyte differentiation, lipid metabolism, and adiponectin secretion ${ }^{49,50}$. Increased amount of adiponectin derived from mature adipocytes is considered as a direct activator of AMPK. A recent study also defined FGF21 as a crucial mediator linking PPAR- $\gamma$ to AMPK ${ }^{51}$. Additionally, thiazolidinediones, agonists of PPAR- $\gamma$, have been proven to be effective in activating $\mathrm{AMPK}^{25,26}$. Collectively, these findings further verify our result that PPAR- $\gamma$ is essential for the activation of AMPK by RA.
In summary, the present study indicated that supplementation of RA attenuated cardiac dysfunction, as well as inhibited cardiac fibrosis following long-term pressure overload in vivo, and blocked transdifferentiation of CFs in vitro. The beneficial effects of RA on fibrotic response may be ascribed to its activation of AMPK and inhibition of Smad3. Our research collectively provided RA as a candidate in the prevention and treatment of cardiac fibrosis.

\section{Materials and methods \\ Antibodies and reagents}

RA ( $\geq 98 \%$ purity, as confirmed by high-performance liquid chromatography) was obtained from Shanghai Winherb Medical Co. (Shanghai, China, no. 160603) and solubilized in $0.1 \%$ dimethylsulfoxide (DMSO). TGF- $\beta$ (T7039) and PE (P6126) were purchased from SigmaAldrich (St. Louis, MO, USA). Replication-defective vectors carrying small hairpin RNA against AMPK $\alpha 2$ (shAmpk $\alpha 2$ ) were obtained from Vigene Bioscience (Rockville, MD, USA), and scramble (short hairpin RNA (shRNA)) was from Sigma-Genosys (Spring, TX, USA). The efficacy of shAmpk $\alpha 2$ has been verified by our previous studies ${ }^{45,52}$. Small interfering RNAs (siRNAs) targeting Ppar- $\gamma$ were generated by RiboBio (RiboBio Co., Ltd, Guangzhou, China). Three siRNAs were generated and the one resulting in the most downregulated expression of PPAR- $\gamma$ was used for further studies. Primary antibodies against the following proteins were purchased from Cell Signaling Technology (Danvers, MA, USA): SOD2 (13141s), COX2 (12282), protein kinase B (T-AKT, 4691), P-AKT (4060), p38 mitogen-activated protein kinase (T-P38, 9212P), P-P38 (4511P), extracellular signal-regulated kinase (T-ERK, 4695), P-ERK (4370P), T-AMPK $\alpha$ (2603P), P-AMPK $\alpha$ (2535), acetylCoA carboxylase (T-ACC, 3676P), P-ACC (3661P), TSmad3 (9513S), P-Smad3 (8769) and glyceraldehyde 3phosphate dehydrogenase (GAPDH, 2118). Antibodies for Nrf2 (ab31163), iNOS (ab3523), $\alpha$-SMA (ab5694), $\alpha-$ actinin (ab68167), vimentin (ab92547) and TGF- $\beta 1$ (ab64715) were obtained from Abcam (Cambridge, UK). Anti-Col I (14695-1-AP), anti-Col III (13548-1-AP), 
and anti-CTGF (23936-1-AP) were purchased from Proteintech (Manchester, UK), whereas anti-proliferating cell nuclear antigen (PCNA, sc-7907, 1:200 dilution) and anti-PPAR- $\gamma$ (sc-7196, 1:200 dilution) were purchased from Santa Cruz Biotechnology (Dallas, TX, USA). The GTVision ${ }^{\mathrm{TM}}+$ Detection System/Mo\&Rb reagent for immunohistochemistry was purchased from Gene Technology (Shanghai, China) and Alexa Fluor 488-goat anti-rabbit secondary antibody for immunofluorescence staining was obtained from LI-COR Biosciences (Lincoln, USA). All antibodies were used at a dilution of 1:1000 if not otherwise indicated. The BCA protein assay kit was from Pierce (Rockford, IL, USA). All other chemicals were of analytical grade.

\section{Animals and treatments}

All animals were given humane care in compliance with the Animal Care and Use Committee of Renmin Hospital of Wuhan University, which is also in agreement with the instruction of Guidelines for the Care and Use of Laboratory Animals published by the United States National Institutes of Health (NIH Publication, revised 2011). A blinded manner ran through the entire experimental process all along, including the surgeries and subsequent analyses. The groups of mice were revealed upon destination of the experiment and the data were obtained and analyzed by two independent individuals, respectively, without a prior knowledge of the hypothesis and interventions in this study.

Male C57/B6 mice (age: 8-10 weeks old; BW: $25.5 \pm 2$ g) were purchased from the Institute of Laboratory Animal Science, Chinese Academy of Medical Sciences (Beijing, China). The animals were allowed access to food and drinking water ad libitum and were fed on a 12-h light/ dark cycle with a controlled temperature $\left(20-25^{\circ} \mathrm{C}\right)$ and humidity $(50 \pm 5 \%)$ environment for a period of 1 week before the study commenced. The $\mathrm{AB}$ model was employed to generate pressure overload induced cardiac remodeling as described previously ${ }^{53}$. Briefly, mice were anesthetized with $3 \%$ pentobarbital sodium $(50 \mathrm{mg} / \mathrm{kg}$, Sigma) by intraperitoneal injection and randomly assigned to $A B$ surgery or sham-operated control group. Under general anesthesia, the thoracic aorta was surgically dissected at the second intercostal space after left hemithoracotomy. Then a 27-G blunt needle was placed along the side of the isolated aorta segment, with a 7-0 silk suture tightly tied around the aorta and the overlying needle. The needle was then removed, thus producing severe aortic constriction. Meanwhile, animals assigned to the sham-operated control group underwent the same procedures without actual ligation of the aorta. Temgesic (qd, $0.1 \mathrm{mg} / \mathrm{kg}$ ) was used subcutaneously for postoperative pain relief. One week after operation, after confirmation for the adequate ligation via Doppler analysis, mice were intragastrically administered (09:00 am) with RA ( $100 \mathrm{mg} / \mathrm{kg})$ once daily referring to previous studies $^{54,55}$ or isovolumic vehicle for 7 weeks. Thus, all the mice were divided into four groups randomly: sham +vehicle, sham $+\mathrm{RA}, \mathrm{AB}+$ vehicle, and $\mathrm{AB}+\mathrm{RA}(n=15$ per group). After the experimental period, mice were sacrificed with heart and tibia collected for calculating $\mathrm{HW} / \mathrm{BW}$ and $\mathrm{HW} / \mathrm{TL}$ ratios and elaborating the underlying mechanisms. Mice were orally administered RA $(100 \mathrm{mg} / \mathrm{kg} / \mathrm{d})$ for 3 weeks beginning at one week postsurgery to explore the effect of RA on inflammatory response in vivo. To ascertain whether the beneficial effect of RA on cardiac fibrosis was mediated by AMPK $\alpha$, AMPK $\alpha 2 \mathrm{KO}$ mice were used. The source of AMPK $\alpha 2$ global KO mice has been described previously ${ }^{45}$.

\section{Echocardiography and hemodynamics}

Transthoracic echocardiography was performed by a MyLab 30CV ultrasound (Esaote SpA, Genoa, Italy) with a $10 \mathrm{MHz}$ phased array transducer as previously described $^{56,57}$. Light anesthetized by $1.5 \%$ isoflurane, mice were lightly secured in a warming pad with a shallow left lateral position after the precordium shaved. After imaged with two-dimensional (2D) mode in the parasternal long-axis and/or parasternal short-axis at the level close to papillary muscles, a 2D guided M-mode trace crossing the anterior and posterior wall of the left ventricle was recorded and the morphological parameters of the heart were collected and calculated. Attention was given not to bring excessive pressure to the chest, which could cause bradycardia and deformation of the heart.

Invasive hemodynamic monitoring was performed by cardiac catheterization according to our previous articles $^{56,57}$. In brief, mice was placed on a warmed surgical platform with supine position and the right carotid artery was isolated, exposed. Then a 1.4-French Millar catheter transducer (SPR-839; Millar Instruments, Houston, TX) was inserted into the left ventricle through the isolated carotid artery. And data were analyzed using the PVAN data analysis software.

\section{Histological analysis and immunohistochemistry}

Fixed hearts were dehydrated and embedded in paraffin. In all, 5- $\mu \mathrm{m}$ slices sectioned from middle segment of the heart were performed to HE for the examination of overall morphology and calculation of cardiomyocyte area, and PSR staining was determined for evaluating collagen deposition. Data were analyzed by a digital analysis software (Image-Pro Plus 6.0, Media Cybernetics, Bethesda, MD, USA) with 50 cells per slide analyzed for the detection of the cardiomyocyte area and $>60$ fields per group assessed for the evaluation of fibrosis.

Immunohistochemical staining was determined for further assessing the extent of cardiac fibrosis by 
GTVision $^{\mathrm{TM}}+$ Detection System/Mo\&Rb (GK600710) according to the manufacturer's protocol. Endogenous peroxidase and the nonspecific binding of the antibody were blocked with $3 \%$ hydrogen peroxide for $20 \mathrm{~min}$ and $10 \%$ goat serum for $45 \mathrm{~min}$, respectively, at room temperature. And slices were incubated with the indicated primary antibodies (1:100) overnight at $4{ }^{\circ} \mathrm{C}$ and with GTVision $^{\mathrm{TM}}+$ Detection System/Mo\&Rb reagent for 60 $\min$ at $37^{\circ} \mathrm{C}$. Then, the slices were examined by the light microscopy after being visualized with diaminobenzidin for $2 \mathrm{~min}$ at room temperature.

\section{Western blot and quantitative real-time PCR}

After being extracted from the frozen pulverized left ventricles or cultured cells and quantified, total proteins were prepared for western blot analysis and normalized to the matched total proteins or GAPDH according to our previous study $y^{52}$. Briefly, separated proteins were incubated with the indicated primary antibodies overnight at $4{ }^{\circ} \mathrm{C}$ and with secondary antibodies for $60 \mathrm{~min}$ at room temperature. Nuclear and cytosolic protein fractions were separated using a commercial kit (Thermo Fisher Scientific) according to the manufacturer's protocol. Proteins from cytosolic lysates were normalized to GAPDH, whereas proteins from nuclear lysates were normalized to PCNA.

Isolated total mRNA from hearts and cultured cells was reversely transcribed to complementary DNA (cDNA) with Transcriptor First Strand cDNA Synthesis Kit (Roche (Basel, Switzerland), 04896866001). Transcriptional level of target genes were normalized to Gapdh, and the primers for quantitative real-time PCR are shown in Supplementary Table 3.

\section{Cell culture, treatments, and infection}

Neonatal rat CFs and cardiomyocytes were prepared by the methods according to previous literatures ${ }^{58,59}$. Bromodeoxyuridine $(0.1 \mathrm{mM})$ was employed to inhibit proliferation of $\mathrm{CFs}$ in neonatal rat cardiomyocytes. Cardiomyocytes were seeded into 6- and 24-well plates and cultured in Dulbecco's modified Eagle's medium (DMEM)/F12 (GIBCO, C11995) supplemented with 10\% fetal bovine serum (FBS, GIBCO, 10099) for $48 \mathrm{~h}$. After grown to $70-80 \%$ confluency and serum deprived for $16 \mathrm{~h}$ to synchronize, cardiomyocytes were assigned randomly to incubate with RA $(20 \mu \mathrm{g} / \mathrm{ml})^{21}$ or equal volume of vehicle for $24 \mathrm{~h}$ in the presence or absence of PE $(50 \mu \mathrm{M})$. Eventually, cells were collected for western blot, quantitative real-time PCR, and immunofluorescence staining without awareness of the sample group allocation during the experiment.

The purity of cultured CFs was measured by a negative result of anti- $\alpha$-actinin and positive result of antivimentin via immunofluorescence staining. $\mathrm{CFs}$ in passages 2 and 3 were used for all studies. After cultured in
DMEM/F12 with 15\% FBS for $48 \mathrm{~h}$ and synchronization, CFs were randomly treated with the indicated concentration of RA with or without TGF- $\beta(10 \mathrm{ng} / \mathrm{ml})$ stimulation for $24 \mathrm{~h}$. To investigate the effect of RA on the phosphorylation and nuclear translocation of Smad3, CFs seeded in six-well plates or coverslips were pretreated with RA $(30 \mu \mathrm{g} / \mathrm{ml})$ for $30 \mathrm{~min}$ before addition of TGF- $\beta$ $(10 \mathrm{ng} / \mathrm{ml})$ to the medium and incubation for an additional $1 \mathrm{~h}^{14}$. RA was dissolved in $0.1 \%$ DMSO as a stock solution $(20 \mathrm{mg} / \mathrm{ml})$ and diluted to the desired final concentrations. For evaluating the effect of RA on the proliferation capacity of CFs, cell counting kit (CCK-8; Dojindo Molecular Technologies, Rockville, MD, USA) was used referring to the manufacturer's protocol.

We knocked down the expression of AMPK $\alpha 2$ via shAmpk $\alpha 2$ carried by adenovirus as our previous study ${ }^{52}$. In brief, CFs were infected with shAmpk $\alpha 2$ or shRNA for $4 \mathrm{~h}$ at a multiplicity of infection (MOI) of 50, then rinsed and synchronized with serum-free medium for $16 \mathrm{~h}$ before further studies.

For PPAR- $\gamma$ inhibition, CFs were treated with GW9662 $(10 \mu \mathrm{M})$, a specific antagonist of PPAR $-\gamma$, for $24 \mathrm{~h}$ prior to interventions ${ }^{60}$. Moreover, genetic ablation targeting Ppar- $\gamma$ with small interfering RNA (siPpar- $\gamma$ ) was performed to knockdown the expression of PPAR- $\gamma$ referring to a previous study ${ }^{61}$.

\section{Wound scratch assay of primary CFs}

Wound scratch assay was performed according to a previous study ${ }^{62}$. Briefly, CFs were passaged in 24-well plates and were cultured to form a confluent monolayer, and then, a scratch in the cell monolayer was generated by using a $200 \mu \mathrm{l}$ sterilized micropipette tip. After being gently rinsed with phosphate-buffered saline (PBS) for three times, CFs were immediately treated with RA (30 $\mu \mathrm{g} / \mathrm{ml})$ or vehicle in the presence or absence of TGF- $\beta$ (10 $\mathrm{ng} / \mathrm{ml}$ ) and were cultured with serum-free medium to exclude the influence of cell proliferation. The cells were photographed before and at $12 \mathrm{~h}$ after treatment using inverted microscope (IX51, Olympus, Tokyo, Japan).

\section{Immunofluorescence staining}

Immunofluorescence staining was performed as previously described ${ }^{52,60}$. Briefly, cardiac slices or cell coverslips were fixed with $4 \%$ formaldehyde, permeabilized in $0.2 \%$ Triton X-100, and stained with $\alpha$-actinin (1:100), $\alpha$ SMA (1:100), or T-Smad3 (1:75) after being blocked with $10 \%$ goat serum for $60 \mathrm{~min}$ at $37^{\circ} \mathrm{C}$, and followed with a Alexa Fluor 488-goat anti-rabbit secondary antibody (1:200), after which slices and coverslips were visualized, with 4,6-diamidino-2-phenylindole (DAPI) used for nuclei observation, via a special OLYMPUS DX51 fluorescence microscope. Images were analyzed via Image-Pro Plus 6.0 in a blind manner. 


\section{Statistical analysis}

Results in our study are presented as the mean \pm standard error of the mean (SEM). Differences between two groups were analyzed by unpaired, two tailed-Student's $t$ tests. To evaluate the effects of RA on $A B$, two-way analysis of variance (ANOVA) followed by Bonferroni's post hoc test was used. One-way ANOVA was carried out to compare differences among three or more groups, followed by post hoc Tukey test. $P<0.05$ was considered as an indicator of statistical significance.

\section{Acknowledgements}

This work was supported by grants from National Natural Science Foundation of China (no.: 81270303, 81470516, 81470402, 81500184, and 81700254), the Key Project of the National Natural Science Foundation (no. 81530012), the Research Program from the Science and Technology Department of Hunan Province (no. 2015SK20455), the Fundamental Research Funds for the Central Universities (no. 2042017kf0085 and 2042015kf0073), Natural Science

Foundation of Hubei Province of China.

\section{Author details}

${ }^{1}$ Department of Cardiology, Renmin Hospital of Wuhan University, Wuhan 430060, China. ${ }^{2}$ Cardiovascular Research Institute of Wuhan University, Wuhan 430060, China. ${ }^{3}$ Hubei Key Laboratory of Cardiology, Wuhan 430060, China

\section{Conflict of interest}

The authors declare that they have no conflict of interest.

\section{Publisher's note}

Springer Nature remains neutral with regard to jurisdictional claims in published maps and institutional affiliations.

\section{Supplementary information}

The online version of this article https://doi.org/10.1038/s41419-017-0123-3 contains supplementary material.

Received: 30 August 2017 Revised: 20 October 2017 Accepted: 2 November 2017

Published online: 24 January 2018

\section{References}

1. Hill, J. A. \& Olson, E. N. Cardiac plasticity. N. Engl. J. Med. 358, 1370-1380 (2008)

2. Vriesendorp, P. A. et al. Impact of adverse left ventricular remodeling on sudden cardiac death in patients with hypertrophic cardiomyopathy. Clin. Cardiol. 37, 493-498 (2014)

3. Kong, P., Christia, P. \& Frangogiannis, N. G. The pathogenesis of cardiac fibrosis. Cell. Mol. Life. Sci. 71, 549-574 (2014).

4. Rienks, M., Papageorgiou, A. P., Frangogiannis, N. G. \& Heymans, S. Myocardial extracellular matrix: an ever-changing and diverse entity. Circ. Res. 114 872-888 (2014).

5. Khan, R. \& Sheppard, R. Fibrosis in heart disease: understanding the role of transforming growth factor-beta in cardiomyopathy, valvular disease and arrhythmia. Immunology 118, 10-24 (2006).

6. leronimakis, N. et al. Coronary adventitial cells are linked to perivascular cardiac fibrosis via TGFbeta1 signaling in the mdx mouse model of Duchenne muscular dystrophy. J. Mol. Cell. Cardiol. 63, 122-134 (2013).

7. Nagpal, V. et al. MiR-125b is critical for fibroblast-to-myofibroblast transition and cardiac fibrosis. Circulation 133, 291-301 (2016).

8. Lal, H. et al. Cardiac fibroblast glycogen synthase kinase-3beta regulates ventricular remodeling and dysfunction in ischemic heart. Circulation 130 419-430 (2014)

9. Shi, Y. \& Massague, J. Mechanisms of TGF-beta signaling from cell membrane to the nucleus. Cell 113, 685-700 (2003).
10. Huang, X. R. et al. Smad3 mediates cardiac inflammation and fibrosis in angiotensin II-induced hypertensive cardiac remodeling. Hypertension $\mathbf{5 5}$ 1165-1171 (2010).

11. Zhang, P. et al. AMP activated protein kinase-alpha2 deficiency exacerbates pressure-overload-induced left ventricular hypertrophy and dysfunction in mice. Hypertension 52, 918-924 (2008).

12. Sung, M. M. et al. Resveratrol treatment of mice with pressure-overloadinduced heart failure improves diastolic function and cardiac energy metabolism. Circ. Heart Fail. 8, 128-137 (2015).

13. Qi, H. et al. Activation of AMPK attenuated cardiac fibrosis by inhibiting CDK2 via p21/p27 and miR-29 family pathways in rats. Mol. Ther. Nucleic Acids $\mathbf{8}$, 277-290 (2017).

14. Wu, D. et al. CTRP3 attenuates post-infarct cardiac fibrosis by targeting Smad3 activation and inhibiting myofibroblast differentiation. J. Mol. Med (Berl.) 93, 1311-1325 (2015)

15. Al-Sereiti, M. R., Abu-Amer, K. M. \& Sen, P. Pharmacology of rosemary (Rosmarinus officinalis Linn.) and its therapeutic potentials. Indian J. Exp. Biol. 37 124-130 (1999).

16. Scheckel, K. A., Degner, S. C. \& Romagnolo, D. F. Rosmarinic acid antagonizes activator protein-1-dependent activation of cyclooxygenase-2 expression in human cancer and nonmalignant cell lines. J. Nutr. 138, 2098-2105 (2008).

17. Al-Dhabi, N. A., Arasu, M. V., Park, C. H. \& Park, S. U. Recent studies on rosmarinic acid and its biological and pharmacological activities. EXCLI J. 13, 1192-1195 (2014).

18. Lu, C., Zou, Y., Liu, Y. \& Niu, Y. Rosmarinic acid counteracts activation of hepatic stellate cells via inhibiting the ROS-dependent MMP-2 activity: involvement of Nrf2 antioxidant system. Toxicol. Appl. Pharmacol. 318, 69-78 (2017).

19. Lee, J. et al. Rosmarinic acid as a downstream inhibitor of IKK-beta in TNFalpha-induced upregulation of CCL11 and CCR3. Br. J. Pharmacol. 148, 366-375 (2006).

20. Zhang, M., Yan, H., Li, S. \& Yang, J. Rosmarinic acid protects rat hippocampal neurons from cerebral ischemia/reperfusion injury via the Akt/JNK3/caspase3 signaling pathway. Brain. Res. 1657, 9-15 (2017).

21. Kim, D. S. et al. Inhibitory effects of rosmarinic acid on adriamycin-induced apoptosis in $\mathrm{H} 9 \mathrm{c} 2$ cardiac muscle cells by inhibiting reactive oxygen species and the activations of c-Jun N-terminal kinase and extracellular signalregulated kinase. Biochem. Pharmacol. 70, 1066-1078 (2005).

22. Li, Q. L. et al. Three angiotensin-converting enzyme inhibitors from Rabdosia coetsa. Phytomedicine 15, 386-388 (2008).

23. Ersoy, $\mathrm{S}$, et al. Endothelium-dependent induction of vasorelaxation by Meliss officinalis L. ssp. officinalis in rat isolated thoracic aorta. Phytomedicine $\mathbf{1 5}$, 1087-1092 (2008).

24. Yang, M. D. et al. Rosmarinic acid and baicalin epigenetically derepress peroxisomal proliferator-activated receptor gamma in hepatic stellate cells for their antifibrotic effect. Hepatology 55, 1271-1281 (2012).

25. Konrad, D. et al. Troglitazone causes acute mitochondrial membrane depolarisation and an AMPK-mediated increase in glucose phosphorylation in muscle cells. Diabetologia 48, 954-966 (2005).

26. Osman, I. \& Segar, L. Pioglitazone, a PPARgamma agonist, attenuates PDGFinduced vascular smooth muscle cell proliferation through AMPK-dependent and AMPK-independent inhibition of mTOR/p70S6K and ERK signaling. Biochem. Pharmacol. 101, 54-70 (2016).

27. Martinez-Martinez, E. et al. Leptin induces cardiac fibrosis through galectin-3, mTOR and oxidative stress: potential role in obesity. J. Hypertens. 32 1104-1114 (2014). discussion 1114.

28. Nicoletti, A. \& Michel, J. B. Cardiac fibrosis and inflammation: interaction with hemodynamic and hormonal factors. Cardiovasc. Res. 41, 532-543 (1999).

29. Sanbongi, C. et al. Rosmarinic acid inhibits lung injury induced by diesel exhaust particles. Free Radic. Biol. Med. 34, 1060-1069 (2003).

30. Ghasemzadeh, R. M., Amin, B., Mehri, S., Mirnajafi-Zadeh, S. J. \& Hosseinzadeh, $H$. Anti-inflammatory effects of ethanolic extract of Rosmarinus officinalis $L$. and rosmarinic acid in a rat model of neuropathic pain. Biomed. Pharmacother. 86, 441-449 (2017).

31. Fetoni, A. R. et al. Rosmarinic acid up-regulates the noise-activated Nrf2/HO-1 pathway and protects against noise-induced injury in rat cochlea. Free Radic. Biol. Med. 85, 269-281 (2015)

32. Liu, $Y$, et al. Toll-like receptor 5 deficiency attenuates interstitial cardiac fibrosis and dysfunction induced by pressure overload by inhibiting inflammation and the endothelial-mesenchymal transition. Biochim. Biophys. Acta 1852 2456-2466 (2015) 
33. Kim, H. K., Lee, J. J., Lee, J. S., Park, Y. M. \& Yoon, T. R. Rosmarinic acid downregulates the LPS-induced production of monocyte chemoattractant protein1 (MCP-1) and macrophage inflammatory protein-1alpha (MIP-1alpha) via the MAPK pathway in bone-marrow derived dendritic cells. Mol. Cells 26, 583-589 (2008).

34. Lee, J., Kim, Y. S. \& Park, D. Rosmarinic acid induces melanogenesis through protein kinase A activation signaling. Biochem. Pharmacol. 74, 960-968 (2007).

35. Divakaran, $V$. et al. Adaptive and maladptive effects of SMAD3 signaling in the adult heart after hemodynamic pressure overloading. Circ. Heart Fail. 2, 633-642 (2009)

36. Schmierer, B., Tournier, A. L., Bates, P. A. \& Hill, C. S. Mathematical modeling identifies Smad nucleocytoplasmic shuttling as a dynamic signal-interpreting system. Proc. Natl. Acad. Sci. USA 105, 6608-6613 (2008).

37. Makino, $\mathrm{T}$. et al. Inhibitory effects of rosmarinic acid on the proliferation of cultured murine mesangial cells. Nephrol. Dial. Transplant. 15, 1140-1145 (2000).

38. Osakabe, N. et al. Rosmarinic acid, a major polyphenolic component of Perilla frutescens, reduces lipopolysaccharide (LPS)-induced liver injury in Dgalactosamine (D-GalN)-sensitized mice. Free Radic. Biol. Med. 33, 798-806 (2002).

39. Jin, B. R. et al. Rosmarinic acid suppresses colonic inflammation in dextran sulphate sodium (DSS)-induced mice via dual inhibition of NF-kappaB and STAT3 activation. Sci. Rep. 7, 46252 (2017).

40. Kato, S. et al. Prognostic significance of quantitative assessment of focal myocardial fibrosis in patients with heart failure with preserved ejection fraction. Int. J. Cardiol. 191, 314-319 (2015).

41. Rathod, R. H., Prakash, A., Powell, A. J. \& Geva, T. Myocardial fibrosis identified by cardiac magnetic resonance late gadolinium enhancement is associated with adverse ventricular mechanics and ventricular tachycardia late after Fontan operation. J. Am. Coll. Cardiol. 55, 1721-1728 (2010).

42. Ma, X. et al. Cardiac fibrosis alleviated by exercise training is AMPK-dependent. PLOS ONE 10, e0129971 (2015).

43. Hermida, N. et al. HMGCoA reductase inhibition reverses myocardial fibrosis and diastolic dysfunction through AMP-activated protein kinase activation in a mouse model of metabolic syndrome. Cardiovasc. Res. 99, 44-54 (2013).

44. Lee, J. E. et al. alpha-Lipoic acid attenuates cardiac fibrosis in Otsuka LongEvans Tokushima fatty rats. Cardiovasc. Diabetol. 11, 111 (2012).

45. Ma, Z. G. et al. Asiatic acid protects against cardiac hypertrophy through activating AMPKalpha signalling pathway. Int. J. Biol. Sci. 12, 861-871 (2016).

46. Jayanthy, G., Roshana, D. V., Ilango, K. \& Subramanian, S. P. Rosmarinic acid mediates mitochondrial biogenesis in insulin resistant skeletal muscle through activation of AMPK. J. Cell. Biochem. 118, 1839-1848 (2017).

47. Mishra, R. et al. AMP-activated protein kinase inhibits transforming growth factor-beta-induced Smad3-dependent transcription and myofibroblast transdifferentiation. J. Biol. Chem. 283, 10461-10469 (2008).
48. $\mathrm{Xu}$, L. et al. A protective role of ciglitazone in ox-LDL-induced rat microvascular endothelial cells via modulating PPARgamma-dependent AMPKVNOS pathway. J. Cell. Mol. Med. 19, 92-102 (2015).

49. Lehrke, M. \& Lazar, M. A. The many faces of PPARgamma. Cell 123, 993-999 (2005).

50. Tishinsky, J. M., Ma, D. W. \& Robinson, L. E. Eicosapentaenoic acid and rosiglitazone increase adiponectin in an additive and PPARgamma-dependent manner in human adipocytes. Obes. (Silver Spring). 19, 262-268 (2011).

51. Zhou, Y. et al. Ampelopsin improves insulin resistance by activating PPARgamma and subsequently up-regulating FGF21-AMPK signaling pathway. PLOS ONE 11, e0159191 (2016).

52. Ma, Z. G. et al. Protection against cardiac hypertrophy by geniposide involves the GLP-1 receptor/AMPKalpha signalling pathway. Br. J. Pharmacol. 173, 1502-1516 (2016)

53. Jiang, D. S. et al. IRF8 suppresses pathological cardiac remodelling by inhibiting calcineurin signalling. Nat. Commun. 5, 3303 (2014).

54. Govindaraj, J. \& Sorimuthu, P. S. Rosmarinic acid modulates the antioxidant status and protects pancreatic tissues from glucolipotoxicity mediated oxidative stress in high-fat diet: streptozotocin-induced diabetic rats. Mol. Cell. Biochem. 404, 143-159 (2015).

55. Boonyarikpunchai, W., Sukrong, S. \& Towiwat, P. Antinociceptive and antiinflammatory effects of rosmarinic acid isolated from Thunbergia laurifolia Lindl. Pharmacol. Biochem. Behav. 124, 67-73 (2014).

56. Ma, Z. G. et al. CTRP3 attenuates cardiac dysfunction, inflammation, oxidative stress and cell death in diabetic cardiomyopathy in rats. Diabetologia 60, 1126-1137 (2017)

57. Xu, S. C., Ma, Z. G., Wei, W. Y., Yuan, Y. P. \& Tang, Q. Z. Bezafibrate attenuates pressure overload-induced cardiac hypertrophy and fibrosis. Ppar. Res. 2017, 5789714 (2017).

58. Schorb, W. et al. Angiotensin II is mitogenic in neonatal rat cardiac fibroblasts Circ. Res. 72, 1245-1254 (1993).

59. Toth, A. et al. Targeted deletion of Puma attenuates cardiomyocyte death and improves cardiac function during ischemia-reperfusion. Am. J. Physiol. Heart Circ. Physiol. 291, H52-60 (2006).

60. Ma, Z. G. et al. Piperine attenuates pathological cardiac fibrosis via PPARgamma/AKT pathways. EbioMedicine 18, 179-187 (2017).

61. Vara, D., Morell, C., Rodriguez-Henche, N. \& Diaz-Laviada, I. Involvement of PPARgamma in the antitumoral action of cannabinoids on hepatocellular carcinoma. Cell Death Dis. 4, e618 (2013).

62. Lorenzen, J. M. et al. Osteopontin is indispensible for AP1-mediated angiotensin II-related miR-21 transcription during cardiac fibrosis. Eur. Heart J. 36, 2184-2196 (2015) 